Epidemiology and Infection

cambridge.org/hyg

\section{Original Paper}

TMembers of the ETHICOBOTS consortium: The members of the Ethiopia Control of Bovine Tuberculosis Strategies (ETHICOBOTS) consortium are: Abraham Aseffa, Adane Mihret, Bamlak Tessema, Bizuneh Belachew, Eshcolewyene Fekadu, Fantanesh Melese, Gizachew Gemechu, Hawult Taye, Rea Tschopp, Shewit Haile, Sosina Ayalew, Tsegaye Hailu, all from Armauer Hansen Research Institute, Ethiopia; Rea Tschopp from Swiss Tropical and Public Health Institute, Switzerland; Adam Bekele, Chilot Yirga, Mulualem Ambaw, Tadele Mamo, Tesfaye Solomon, all from Ethiopian Institute of Agricultural Research, Ethiopia; Tilaye Teklewold from Amhara Regional Agricultural Research Institute, Ethiopia; Solomon Gebre, Getachew Gari, Mesfin Sahle, Abde Aliy, Abebe Olani, Asegedech Sirak, Gizat Almaw, Getnet Mekonnen, Mekdes Tamiru, Sintayehu Guta, all from National Animal Health Diagnostic and Investigation Centre, Ethiopia; James Wood, Andrew Conlan, Alan Clarke, all from Cambridge University, UK; Henrietta L. Moore and Catherine Hodge, both from University College London, UK. Constance Smith at University of Manchester, UK; R. Glyn Hewinson, Stefan Berg, Martin Vordermeier, Javier Nunez-Garcia, all from Animal and Plant Health Agency, UK; Gobena Ameni, Berecha Bayissa, Aboma Zewude, Adane Worku, Lemma Terfassa, Mahlet Chanyalew, Temesgen Mohammed, Yemisrach Zeleke, all from Addis Ababa University, Ethiopia.

*These authors contributed equally to this work and should be considered joint first authors.

Cite this article: Mekonnen GA et al (2021). Dynamics and risk of transmission of bovine tuberculosis in the emerging dairy regions of Ethiopia. Epidemiology and Infection 149, e69, 1-9. https://doi.org/10.1017/

S0950268821000480

Received: 23 September 2019

Revised: 8 January 2021

Accepted: 17 February 2021

\section{Key words:}

Apparent prevalence; bovine tuberculosis; emerging dairy belts; Ethiopia; incidence rate; positive reactors removal; risk factors

\section{Author for correspondence:}

G. A. Mekonnen,

E-mail: getnet.abie.mekonnen@gmail.com

(c) The Author(s), 2021. Published by Cambridge University Press. This is an Open Access article, distributed under the terms of the Creative Commons AttributionNonCommercial-NoDerivatives licence (http:// creativecommons.org/licenses/by-nc-nd/4.0/), which permits non-commercial re-use, distribution, and reproduction in any medium, provided the original work is unaltered and is properly cited. The written permission of Cambridge University Press must be obtained for commercial re-use or in order to create a derivative work.

\section{CAMBRIDGE UNIVERSITY PRESS}

\title{
Dynamics and risk of transmission of bovine tuberculosis in the emerging dairy regions of Ethiopia
}

G. A. Mekonnen ${ }^{1,2, \star}$ (D) A. J. K. Conlann ${ }^{3, \star}$, S. Berg ${ }^{4}$, B. T. Ayele ${ }^{5}$, A. Mihret $^{6}$, A. Olani ${ }^{1}$, H. Asgedom ${ }^{1}$, The ETHICOBOTS consortium ${ }^{1, \dagger}$, J. L. N. Wood ${ }^{3}$ and G. Ameni2,7
${ }^{1}$ National Animal Health Diagnostic and Investigation Centre, P.O. Box 04, Sebeta, Ethiopia; ${ }^{2}$ Aklilu Lemma Institute of Pathobiology, Addis Ababa University, P. O. Box 1176, Addis Ababa, Ethiopia; ${ }^{3}$ Disease Dynamics Unit, Department of Veterinary Medicine, University of Cambridge, Madingley Road, Cambridge CB3 OES, UK; ${ }^{4}$ Bacteriology Department, Animal and Plant Health Agency, Weybridge, Surrey KT15 3NB, UK; ${ }^{5}$ Division of Epidemiology and Biostatistics, Faculty of Medicine and Health Sciences, Stellenbosch University, Stellenbosch, South Africa; ${ }^{6}$ Armauer Hansen Research Institute, P.O. Box 1005, Addis Ababa, Ethiopia and ${ }^{7}$ Department of Veterinary Medicine, College of Food and Agriculture, United Arab Emirates University, P. O. Box: 15551, Al Ain, UAE

\section{Abstract}

The Ethiopian government has several initiatives to expand and intensify the dairy industry; however, the risk of bovine tuberculosis (bTB) spread is a challenge. To assess the rate of expansion and risk factors for transmission of bTB within-herds, we carried out a repeated cross-sectional survey at two time points, 2016/17 and 2018, in three regional cities, namely, Gondar, Hawassa and Mekelle, representing the emerging dairy belts of Ethiopia. The total number of herds involved was 128, comprising an average of 2303 cattle in each round. The Single Intradermal Comparative Cervical Tuberculin (SICCT) test was used to identify reactor status and data on herd-level risk factors were collected using a structured questionnaire. In the first survey, the apparent prevalence of bTB, as measured by the SICCT test, was $4.5 \%$ (95\% CI 3.7-5.4\%) at the individual animal-level and $24 \%$ (95\% CI $17.5-32 \%)$ at the herd-level. There was no statistically significant change in the overall apparent prevalence or regional distribution at the second survey, consistent with the infection being endemic. The incidence rate was estimated at 3.6 (95\% CI 2.8-4.5) and 6.6 (95\% CI 3.0-12.6) cases/ 100 cattle (or herd)-years at the animal- and herd-levels, respectively. Risk factors significantly associated with the within-herd transmission of bTB were age group and within-herd apparent prevalence at the start of the observation period. We noted that farmers voluntarily took steps to remove reactor cattle from their herds as a consequence of the information shared after the first survey. Removal of reactors between surveys was associated with a reduced risk of transmission within these herds. However, with no regulatory barriers to the sale of reactor animals, such actions could potentially lead to further spread between herds. We therefore advocate the importance of setting up regulations and then establishing a systematic bTB surveillance programme to monitor the impact prior to implementing any control measures in Ethiopia.

\section{Introduction}

Bovine tuberculosis (bTB) is a chronic disease of livestock caused mainly by Mycobacterium bovis (M. bovis), with $M$. bovis infection also a risk to wildlife and humans. bTB is important economically, with impacts on both animal productivity and international trade [1-6]. The importance of the disease has been increasing in developing countries where bTB is poorly controlled [7-9]. In some industrial countries, bTB control in cattle has been achieved or at least the disease burden markedly reduced through the implementation of test and slaughter policies [10]. In some contexts, notably in the UK, Ireland and New Zealand, significant wildlife reservoirs contribute to disease spillover back to domesticated animals, hampering progress towards elimination [11]. In Ethiopia, studies conducted so far have demonstrated that bTB is endemically established in its cattle population; however, there are no known wildlife reservoirs and the distribution of the disease is heterogeneous. Pastoral and crop-livestock production systems holding primarily Zebu cattle have lower animal prevalence $(<10 \%)[12-16]$ than herds in urban and peri-urban areas where genetically improved dairy cattle, mainly Holstein Friesian - Zebu crosses, are managed under intensive and semi-intensive management systems. These intensively managed herds have a higher prevalence and are mainly found in the central part of Ethiopia (11-47\% animal prevalence) [17-20]. An important driving factor for the spread of bTB is unregulated cattle movement/trading with poor biosecurity measures 
following the Ethiopian government initiatives and plans to expand and intensify the dairy industry in the country [21]. Dairy farming is an emerging sector in the peripheral regions of the country, particularly in regional cities aiming to satisfy the demand for milk and milk products. The demand for dairy cattle in these areas is fulfilled by trade in areas where dairy farming is relatively well developed, both locally and in remote areas, but without prior knowledge as to the bTB status of the animals. Thus, the risk of spread of bTB and other production-related cattle diseases has become the constraining factor for the expansion of the industry.

The economic and public health risks of bTB in Ethiopia are assumed to be significant. For intensive dairy herds in Addis Ababa, infection with bTB has been estimated to cause a financial loss of 4-6\% per year [22]. Condemnation of bTB-affected carcasses in slaughterhouses can also have an economic impact on the livelihoods of livestock keepers in Ethiopia [23]. In humans, the total incidence rate of tuberculosis in Ethiopia was 151 (107-204) per hundred thousand population in 2018 [24]. The prevalence of human tuberculosis due to $M$. bovis has been reported at $2.8 \%$ of all human tuberculosis cases globally, corresponding to about seven zoonotic tuberculosis cases per hundred thousand population per year [25]. In Ethiopia, although several publications have reported a relatively low prevalence of zoonotic tuberculosis, it is possible that the prevalence is higher among high-risk populations directly exposed to bTB-infected cattle or to unpasteurised dairy products [26-28]. The need for controlling $\mathrm{bTB}$ to reduce the zoonotic risk and economic loss is, therefore, pressing.

Currently, there is no national bTB control programme for cattle in Ethiopia. Some intermittent attempts by individual farmers through voluntary removal or segregation of reactor cattle have been reported [29]. Surveillance without statutory control on reactor animals has, in the view of the authors, become a challenge in the Ethiopian condition. Some farmers may be tempted to sell reactor cattle (G.A. Mekonnen, personal observation). This poses the risk of increasing bTB spread/transmission to other herds. Imposing veterinary regulatory procedures to reduce the risk of transmission is currently being considered by the Ethiopian government. In the present study, we conducted repeated tuberculin skin testing on herds in three regions of Ethiopia. We set out to assess the extent to which apparent prevalence is stable in the emerging dairy belts, to quantify the risk factors associated with transmission within herds using the subset of animals that became test-positive in the second test that were negative in the first test, and to assess the response of farmers to being informed of the reactor status of animals within their herds.

\section{Materials and methods}

\section{Study sites}

The study was conducted in three regional administrative cities, namely, Hawassa, Gondar and Mekelle in the southern, northwestern and northern parts of Ethiopia, respectively. This choice was informed by the Ethiopian government strategic plan for dairy development, the three cities chosen to be representative geographically and of the modern dairy industry managed under intensive and semi-intensive systems. These cities are considered emerging dairy belts with relatively large dairy cattle populations (Table 1), and human populations of around 300000 each [30].
Table 1. Dairy herds and cattle recruited for the 2016/17 and 2018 testing scheme

\begin{tabular}{cccc}
\hline & & \multicolumn{2}{c}{$\begin{array}{c}\text { Number tested (\% from } \\
\text { the total) }\end{array}$} \\
\cline { 3 - 4 } Site/Level & Total number per site & $2016 / 17$ & 2018 \\
\hline Gondar & & & \\
\hline Herd & 440 & $59(13.4)$ & $42(9.5)$ \\
\hline Animal & 4800 & $976(20.3)$ & $711(14.8)$ \\
\hline Hawassa & & $54(27)$ & $40(20)$ \\
\hline Herd & 200 & $960(18.5)$ & $972(18.7)$ \\
\hline Animal & 5200 & $61(23.5)$ & $46(18)$ \\
\hline Mekelle & & & $634(24.4)$ \\
\hline Herd & 260 & $174(19.3)$ & $128(14.2)$ \\
\hline Animal & 2600 & $2756(21.9)$ & $2317(18.4)$ \\
\hline Total & 900 & & \\
\hline Herd & 12600 & & \\
\hline Animal & & & \\
\hline & & & \\
\hline
\end{tabular}

\section{Study population}

The study population was dairy cattle managed in the selected herds at the study sites. The dairy cattle (cattle reared for the purpose of milk production) in the selected herds were study units. The majority of animals were crosses of Holstein, Friesian and Zebu breeds (96.4\%), with a few crosses of Jersey and Zebu $(1.9 \%)$, or pure Zebu (1.7\%). The management and setting of farms differed depending on the farmers' experience and level of knowledge of dairy farming.

\section{Study design and sampling strategy}

The study design was cross-sectional with skin testing being repeated at a 1-2 years interval. The first round of testing was carried out across the years 2016-2017 and all tests in the second round were carried out within 2018. The sampling strategy was a one-stage cluster design, considering dairy herds as clusters and individual cattle as sampling units. Sample size calculations were described previously [31]. The number of dairy herds computed and the corresponding number of cattle within the herds are shown in Table 1 . During the first round of testing in 2016/17, herds with more than 20 animals were selected without any prerequisite, while herds with fewer than 20 animals were recruited using a random selection method among the complete list of dairy herds in the area. In 2018, every possible effort was made to retest the same herds selected in 2016/17. However, only a subset of herds could be retested as some refused to participate and a number of herds with high bTB burden were dissolved or changed to other types of businesses. In total, $26 \%(46 / 174)$ of the herds tested in the first survey were not included in the retest. To easily identify individual cattle, in the second round of testing, six digit ear tags were applied as an identifier for all cattle that had no owner-provided ear tag during the first survey. In both rounds of testing, all cattle except calves younger than 4 weeks, clinically sick cattle with disease not suggestive of bTB and cows in the last month of pregnancy were included. These inclusion criteria were 
set to avoid possible interference with the action of tuberculin [8, 32]. During the first round of survey, farmers were all informed of the reactor status of their cattle and relevant advice was forwarded on how to reduce the risk of bTB transmission such as removal of reactor cattle, avoiding contact with neighbour herds or new cattle of unknown bTB status.

\section{Skin testing}

The Single Intradermal Comparative Cervical Tuberculin (SICCT) test method was used to determine the bTB reactor status of cattle. The test was applied as described previously [31]. Briefly, two sites in the middle third of the neck were shaved $12-15 \mathrm{~cm}$ apart parallel to the shoulder and the skin fold thickness measured. Animals were then inoculated with $0.1 \mathrm{ml}(2500$ $\mathrm{IU} / \mathrm{ml})$ avian PPD and $0.1 \mathrm{ml}(3000 \mathrm{IU} / \mathrm{ml})$ bovine PPD (Lelystad B.V., The Netherlands) in the prepared sites. The status of the tuberculin reaction was examined and the skin thickness was measured $72( \pm 4) \mathrm{h}$ after the first injection. A reaction was considered positive if the increase in skin thickness at the bovine site of injection was more than $4 \mathrm{~mm}$ greater than the reaction shown at the site of the avian injection, inconclusive if the increase was from 1 to $4 \mathrm{~mm}$, or negative if the increase was $<1 \mathrm{~mm}$ [33].

\section{Questionnaire survey}

Information on herd structure and risk factors was collected during the retesting in 2018 using a modified questionnaire employed previously [31]. Risk factors considered in this study were identified from the literature $[5,19]$; a complete list of these factors was published [31], a subset of the risk factors that were assumed to have association was included in this survey. The questionnaire contained open-ended and closed questions and was filled in by one researcher in all sites. Administration of the questionnaire was made using local language and in a way that the respondents felt easy. The objective and possible outcome of the study were explained and the respondents were told they could terminate the interview at any stage. As part of the survey, the bTB testing team treated sick animals with antibiotics, anthelminthic drugs and wound spray as incentives and advised owners to seek further advice from the local veterinary services.

\section{Ethical considerations}

Ethical approval to implement the research was granted by the Institutional Review Board (IRB) of Aklilu Lemma Institute of Pathobiology, Addis Ababa University (Reference number IRB/ ALIPB/2018). This study was supported by the Ethiopian Ministry of Agriculture.

\section{Data analysis}

$\mathrm{R}$ statistical software (version 3.5.1) (R Core Team) and RStudio were used for all data analyses and modelling. SICCT test results and risk factor data collected from the subset of herds that were tested twice were examined. Apparent prevalence was calculated using the proportion of test-positives among all tested herds or animals, while the incidence rate between the two tests was defined as the number of cases (new test positive cattle or herds) per 100 cattle (or herd)-years. Time at risk, as measured herd-years required for herd-level incidence rate calculation, was the average follow-up period of the cattle in the herds. For the animal-level incidence rate, the animal-year was the summation of the time intervals between the two SICCT tests of the retested animals and the age of all calves born between surveys. In the incidence rate estimation, new infections were assumed to occur at the midpoint of the follow-up period.

The sub-group of animals with matched test results was used to explore individual animal risk factors for transmission in the interval between tests using generalised linear mixed models (GLMM) with a binomial response (logit link) and estimated using maximum likelihood (Adaptive Gauss-Hermite Quadrature) using the 'Ime4' package [34]. Risk factors for inclusion in the final multivariable model were selected using a univariable screen, retaining variables with $P<0.20$.

We estimated a herd-level random intercept to adjust for the heterogeneity in transmission risk between herds. Given the variability in apparent prevalence both within and between regions, we first considered a nested random effect (herd nested within the region). However, this model is singular and following Barr et al. [35], we simplified the model to include only a herd-level random effect. The random effect was tested by comparing the likelihood ratios of the models with and without the random effects and the difference was confirmed to be significant $(P<$ $0.001)$ by the $\chi^{2}$ test [36]. The variance inflation factor was calculated to check the multicollinearity terms of the model using the package 'performance' [37]. Model fit was assessed by Hosmer and Lemeshow goodness of fit test using 'resourceSelection' package [38]. Finally, the classification capability of the model was checked by the receiver operating characteristic curve (ROC) using ' $P R O C$ ' package [39]. Package 'aod' [40] and 'questioner' [41] were used to calculate the odds ratio (OR) and confidence intervals (CIs) in the GLMM and GLM, respectively. CIs for apparent prevalence and incidence rate were calculated using 'EpiTools epidemiological calculators' with Wilson method [42] and 'OpenEpi' with Fisher's exact test [43], respectively. In all cases, a $95 \%$ confidence level and a significance level of $5 \%$ were used to determine statistical significance.

\section{Results}

The apparent prevalence of bTB as measured by the SICCT test for our matched herds is summarised in Table 2, stratified by region and round of testing. A total of 128 herds were tested twice. The numbers of cattle tested in the two rounds were more or less similar, with 2294 cattle tested in 2016/17 and 2317 in 2018 . This reflects the balance between the number of cattle removed between the two time points and the number of calves born $(n=624)$ and newly introduced $(n=194)$.

GLM regression analysis was done to assess if herds not included in the retest $(26 \%, 45 / 174)$ were associated with their SICCT test reactor status disclosed in the first test. The model output showed that herds reduced from the retest due to refusal to participation or dissolved herds or change of farming businesses were not statistically significantly associated with the bTB positivity (OR $0.63,95 \%$ CI $0.25-1.7, P>0.05$ ). At the animallevel, 35\% (802/2317) of the animals tested in 2018 could not be paired with animals tested in 2016/17 due to lost or changed ear tags. To assess the unpaired rate (assumed to mirror the removal rate) between reactors and non-reactors classified by the first test, a regression analysis using GLMM with herd ID as a random effect was done by adjusting with age to account for the background removal rate. The model output showed that the removal rate in the reactor group of animals was three 


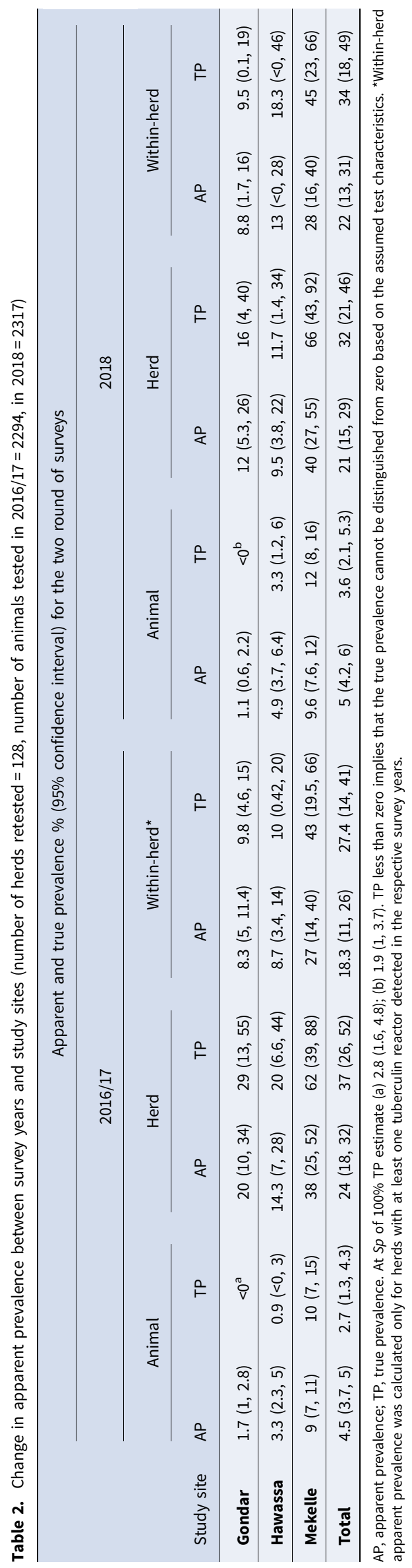

times higher compared to the removal rate in non-reactors (OR 3.0, 95\% CI 1.4-7). However, both the animal and herd-level apparent prevalence were consistent between the two surveys, with no significant difference between the two time points and a consistent (and statistically significant) difference between the study sites.

\section{Incidence rate}

Eight hundred and eighty-six animals belonging to 107 herds were matched between the 2016/17 and 2018 SICCT herd tests using ear tags. The majority of matched animals were SICCT-negative in both tests $(n=810), 15$ cattle were tested positive in both surveys, 57 became positive in the second test, and four were positive in the first test but tested negative in the second SICCT test. These four animals were all relatively strong reactors in the first test (bovine PPD measurements of 9-22 mm, Table 3). Two would have been classified as reactors under the single intradermal tuberculin test with a skin thickness increase $>4 \mathrm{~mm}$, but all four demonstrated a considerable reduction in their response to bovine PPD of $\sim 10 \mathrm{~mm}$ between the two tests. To estimate the overall incidence rate at animal-level, a subset of paired cattle that were SICCT test-negative in the first survey $(n=867)$ and calves born between the two surveys $(n=624)$ were used to estimate the incidence rate of bTB positivity and assess the risk of transmission within herds. Accordingly, the animal-level incidence rate was estimated at 3.6/100 animal-years (Table 4). The incidence rate in Mekelle and Hawassa was significantly higher compared to that in Gondar $(P<0.05)$. The higher incidence of bTB positivity in Mekelle suggested a higher risk of within-herd transmission as would be expected given the higher apparent prevalence of reactors in the first test. In Hawassa, although the overall apparent prevalence in the 2016/17 survey was lower (see Table 2), an overall higher incidence rate was observed due to the contribution of a single large farm $(n=113)$. Although this farm attempted to segregate or remove reactor animals identified in the first round, there was a twofold increase in the within-herd apparent prevalence $(21 \%, 24 / 113$, first survey to $47 \%, 68 / 145$, retest).

At the herd-level, 24\% (31/128, 95\% CI 17.5-32\%) of the retested herds were positive in the first survey, while the remaining were classified negative by the SICCT test. Out of the negative herds in the 2016/17 test, the herd-level incidence rate of bTB positivity was 6.6 (95\% CI 3.0-12.6) per 100 herd-years (Table 4).

\section{Risk factors associated with bTB transmission}

The detection of animals switching from negative to positive SICCT status between tests provides an opportunity to explore risk factors for transmission over this period. GLMM was used to adjust for clustering effects within herds. The model fit and classification capability of the GLMM were evaluated as described above (methods). The Hosmer and Lemeshow goodness of fit revealed no evidence of a systematic lack of fit of the model $\left(\chi^{2}=7.76, \mathrm{df}=8, \quad P=0.46\right)$. The classification ability of the model as evaluated by ROC analysis is excellent (area under the curve 0.96). Exposure time, age group, herd size, herd risk and sex were selected by the univariable screen. Herd risk was categorised based on the within-herd apparent prevalence according to the SICCT test result of the 2016/17 survey. Sex was dropped from the final model due to the small number of males, which were normally removed from the herd by the owners at an early age to reduce maintaining cost. The univariable screen 
Table 3. Skin test reading of animals that were positive by first survey becoming negative in the second survey

\begin{tabular}{lrrrrrrr}
\hline & \multicolumn{3}{c}{$2016 / 17$ survey } & & \multicolumn{3}{c}{2018 survey } \\
\cline { 2 - 4 } \cline { 6 - 8 } $\begin{array}{l}\text { Animal } \\
\text { ID }\end{array}$ & $\Delta \mathrm{B}$ & $\Delta \mathrm{A}$ & $\Delta \mathrm{BA}$ & & $\Delta \mathrm{B}$ & $\Delta \mathrm{A}$ & $\Delta \mathrm{BA}$ \\
\hline $7 \mathrm{~A} 0118$ & 10.45 & 5.33 & 5.12 & 0.09 & 0.69 & -0.6 \\
\hline $7 \mathrm{~A} 0615$ & 9.13 & 3.52 & 5.61 & 0.93 & 2.88 & -1.95 \\
\hline $8 \mathrm{~A} 0404$ & 22.87 & 6.66 & 16.21 & 6.74 & 7.1 & -0.36 \\
\hline $8 \mathrm{~A} 0312$ & 13.82 & 6.66 & 7.16 & 5.67 & 5.51 & 0.16 \\
\hline
\end{tabular}

$\Delta$ - Greek letter delta; $\Delta \mathrm{A}$ and $\Delta \mathrm{B}$ - difference in measurements at time 0 and $72 \mathrm{~h}$ post-PPD injection of the avian PPD and bovine PPD, respectively; while $\triangle B A$ stands for the difference of the measurement increases at the bovine and avian sites after $72 \mathrm{~h}$ post-injections.

also identified calves born in between the surveys as having a lower risk of being a reactor (OR 0.4, 95\% CI 0.2-0.7). However, this variable was omitted from the final multivariable model to avoid its confounding effect with age.

The estimated risk factors from the final multivariable model are presented in Table 5. Age and herd risk were the only explanatory factors to have a significant association with the risk of becoming a reactor, while exposure time and herd size had a large effect size but were not statistically significant. The pattern of incidence of SICCT test positivity across age groups appeared to increase with the peak risk between the age of 5 and 7 years.

\section{Farmers' response to disclosure of reactor status of their cattle}

Following the first survey in 2016/17, farmers were advised to remove reactor cattle to reduce transmission and clearing herds of bTB through time. SICCT test results of the 2018 survey comparing herds that removed reactors and herds that did not remove reactors are summarised in Table 6. Among the retested herds in the 2018 survey $(n=128), 31$ were tested positive in $2016 / 17$ : of these, reactor cattle were removed from 18 herds following disclosure of reactor status; while no reactors were removed from 13 herds.

In herds where reactors were removed, the average within-herd apparent prevalence was decreased by $75 \%$ (within-herd apparent prevalence in 2016/17, 9.3\% (95\% CI 6.7-11.9); in 2018, 2.3\% (95\% CI 0-4.7)); however, in herds where reactors were partially or totally not removed, the average within-herd apparent prevalence increased marginally by $8.3 \%$ (within-herd apparent prevalence in $2016 / 17,25.3 \%$ (95\% CI 12.2-38.4); in 2018, 27.4\% (95\% CI 11.3-43.5)).
Considering only the sub-population of cattle that were SICCT test-negative in the 2016/17 survey (and calves born in between survey years), the incidence rate in herds that did not remove reactors was higher (16.6 per 100 animal-years, animal-years 259) compared to herds that removed reactors (3 per 100 animalyears, animal-years 365 ) following the disclosure of reactor status of the cattle. This corresponds to an $82 \%$ difference in the incidence rate on these herds compared to those that did not remove reactors.

\section{Discussion}

In Ethiopia, a lower apparent prevalence of bTB has been reported in dairies in peripheral regions as compared to the highly infected dairy belts in central Ethiopia [20], and this raises the question as to whether these populations are endemically infected at a lower level than the dairy herds in central Ethiopia or these herds are merely at an earlier stage of infection. To address this question, we carried out a second round of testing on herds previously screened for bTB to assess how apparent prevalence within the herds changed and to identify risk factors associated with the detection of new reactor animals. As the status of animals was disclosed to farmers during the first round of testing, we also had an opportunity to assess the effect of farmers' response to this knowledge and the impact this had on the risk of transmission within their herds.

\section{Herd- and animal-level apparent prevalence and incidence rate}

In this study, there was no statistically significant difference in the overall apparent prevalence of bTB between surveys. Despite the active steps taken to remove reactors from some herds, which could have caused some level of selection bias, the loss of reactor animals was effectively balanced by the detection of new reactors on the second test. While this is suggestive of an endemically infected population, the rate of increase in apparent prevalence may simply be too slow to detect given the relatively short period between tests and the size of our sample.

Comparing between study sites, there were some intriguing regional differences and outlier herds that warrant further discussion. The differences in apparent prevalence between study sites were consistent between the two surveys, with apparent prevalence in Mekelle still significantly higher than the other two regions. In line with this difference in apparent prevalence, the number of new reactors in the second round of testing was higher

Table 4. Estimated incidence rate of bTB reactors based on the number of new reactors in 2018 among herds and animals

\begin{tabular}{|c|c|c|c|c|c|c|c|c|}
\hline \multirow[b]{2}{*}{ Study site } & \multicolumn{4}{|c|}{ Herd } & \multicolumn{4}{|c|}{ Animals } \\
\hline & $n$ & Case & Herd-years & $\begin{array}{c}\text { Incidence rate/100 } \\
\text { herd-years }\end{array}$ & $n$ & Case & Animal-years & $\begin{array}{c}\text { Incidence rate/100 } \\
\text { animal-years }\end{array}$ \\
\hline Gondar & 35 & 1 & 34.6 & $2.9(0.1,15)$ & 444 & 5 & 449 & $1.1(0.4,2.6)$ \\
\hline Hawassa & 34 & 1 & 45.5 & $2.2(0.06,12)$ & 594 & 34 & 779 & $4.3(3,5.9)$ \\
\hline Mekelle & 29 & 6 & 41 & $14.6(5.6,29)$ & 453 & 30 & 688 & $4.4(3,6.2)$ \\
\hline Total & 98 & 8 & 121 & $6.6(3,12.6)$ & 1491 & 69 & 1916 & $3.6(2.8,4.5)$ \\
\hline
\end{tabular}

Herd-years - calculated using average follow-up year(s) of the animals in the herds (Gondar - 1 year, Hawassa - 1.37 years and Mekelle - 1.57 years); animal-years - summation of the time interval between the two SICCT tests for the retested cattle and age of calves born between surveys were considered; new infection was assumed to occur at the mid of the follow-up period and this was considered in the calculations; $n$ - stands for number of herds or animals under investigation. Herds and animals considered for incidence rate estimation were all SICCT-negative in the 2016/17 test. 
Table 5. Risk factors from multivariable GLMM model for the incidence of bTB reactor status (number of animal = 1491; number of herds $=128 ; 2$ missing on animal data)

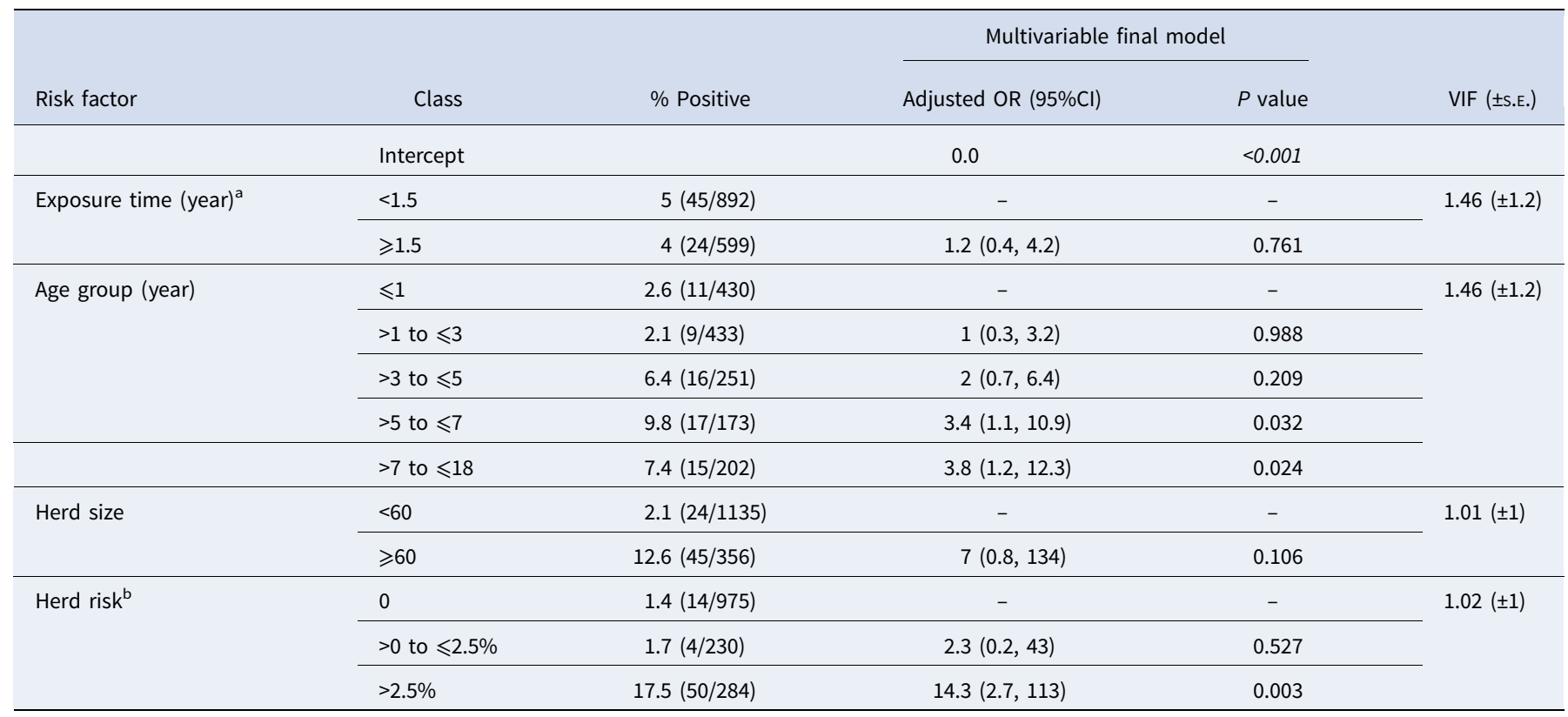

OR, odds ratio; VIF, variance inflation factor; s.E., standard error.

${ }^{a}$ Exposure time refers to the time period the animals/herds were followed.

${ }^{\mathrm{b}}$ Herd risk was categorised based on the within-herd apparent prevalence according to the SICCT test status from the 2016/17 survey (category 1: herds with 0 apparent prevalence, category

2: herds with apparent prevalence between $0 \%$ and $2.5 \%$, category 3 : herds with apparent prevalence of $>2.5 \%$ ).

Table 6. Comparison of herd and animal-level bTB apparent prevalence following removal of SICCT test reactor cattle from herds that were positive in the 2016/17 test (number of herds $=31$, number of animals $=751$ )

\begin{tabular}{lccc} 
& \multicolumn{2}{c}{ Number of herds (animals) } & \\
\cline { 2 - 3 } Control practice & SICCT-positive & SICCT-negative & Total \\
\hline $\begin{array}{lccc}\text { Reactor removed } \\
\text { Reactors not }\end{array}$ & $6(20)$ & $12(428)$ & $18(448)$ \\
\hline Total & $11(71)$ & $2(232)$ & $13(303)$ \\
\hline
\end{tabular}

in Mekelle and Hawassa than in Gondar. This could simply be related to the presence of a higher proportion of infected herds and animals with little or no control effort being done following the disclosure of the 2016/17 SICCT test results.

In Hawassa, a higher animal-level incidence rate of SICCT test positivity suggests a higher rate of within-herd transmission from reactors that were not removed from the herds. The difference was driven by a single large herd $(n=113)$, which attempted to introduce control through the segregation of reactor animals. However, segregation was poorly achieved, which might have contributed to the twofold increase in the number of reactors over the course of a single year. Historical data for this specific herd showed that the herd was first infected following a new cattle introduction in the period between 2014 and 2015 (NAHDIC, unpublished data). Unlike the experience in this herd, proper segregation practice has been suggested to reduce the incidence rate of reactor status. In this regard, Ameni et al. [29] reported a reduction of incidence from 14 cases to one case/100 cattle/year after three consecutive test-and-segregation exercises. However, the practicality of this strategy in Ethiopia may be limited as it highly depends on the availability of sufficient space and commitment to completely isolate bTB reactors from SICCT test-negative animals.

\section{Risk factors for transmission within herds}

A key motivation for the repeated testing of our study herds was to attempt to quantify the rate of transmission of bTB and associated risk factors to inform mathematical transmission modelling of control. To this end, we carried out a risk factor analysis using a specific dataset of cattle negative in the 2016/17 survey and calves born in between the surveys to identify risk factors associated with the detection of new reactor animals. We found that age group and herd risk were significantly associated with animals becoming reactors to the SICCT test.

The present findings and others [44-47] suggest age affects the probability that an animal tests positive and transmission risk increases with age due to a higher probability of contact and/or prolonged exposure to other infected animals or environmental contamination. The magnitude of the within-herd bTB apparent prevalence in the first test increased the risk of animals becoming reactors in the second survey. Cattle kept in herds with apparent prevalence $>2.5 \%$ were more likely to be reactors in the second survey than those kept in negative herds or herds with apparent prevalence below $2.5 \%$ (Table 5). This is probably due to the presence of a larger number of infected animals in these herds serving as sources of infection for non-infected animals, the rate of which can indeed be facilitated in poorly managed herds $[48,49]$.

However, these results must be carefully interpreted with respect to key limitations of our study and the quality of the data we were able to collect. The SICCT test has a relatively high specificity [50], but low sensitivity $[8,51,52]$, so the true prevalence of bTB is likely to be considerably higher than the apparent prevalence. Furthermore, new reactor animals identified 
in the second round may include animals missed by the first round of testing (specifically cattle in an early stage of infection, intercurrent, especially parasitic infections, and/or temporary anergic state like recent parturition during the first survey), thus increasing the apparent rate of transmission between animals. The use of ancillary test methods, such as the interferon $\gamma$ test in combination with the SICCT test could have increased sensitivity (albeit at the expense of specificity) [53]. This was unfortunately not possible in this study due to the remoteness of the study populations from suitable laboratory facilities, a key challenge in the context of less developed countries.

Another important limitation of our dataset is the potential that selection bias has been introduced in our sample of matched animals/herds between tests. We were only able to pair about $52 \%$ of cattle present in the first round during the second round of testing, despite efforts to ensure the identifiability of animals. We applied ear tags to all cattle that had no identification (ID) in the first round of testing. However, some of these IDs were replaced by farmers between visits by a new national ID introduced by the Ethiopian government to establish a national-level dairy database. IDs used by farmers (cattle names) were found not to be reliable and prone to change when animal attendants leave the farm as no written records were kept. Such problems are expected to be a challenge in developing countries, particularly in situations where there is no national identification and traceability system. With regards to the SICCT test, the interpretation of the results used in this study was according to the recommended OIE standard method [33]; however, the estimate for animal-level apparent prevalence is likely imperfect due to the considerable uncertainty of the test sensitivity and specificity for bTB diagnosis. Sensitivity and specificity for the SICCT test in Ethiopian herds have been estimated as 59\% (95\% CI 49-69\%) and $97 \%$ (95\% CI 89-100\%), respectively [54]. The estimate for animal-level true prevalence in the first and second rounds of testing using the point estimates from this study was $2.7 \%$ (95\% CI $1.3-4.3 \%$ ) and $3.6 \%$ (95\% CI $2.1-5.3 \%$ ), respectively (Table 2), slightly lower than the apparent prevalence suggesting a relatively lower specificity of the test. The negative 'true prevalence' estimates in Gondar for animal-level prevalence are an artefact of the sample size and small number of affected herds - with high confidence that the true within-herd prevalence in this area is great than zero. Estimates of true prevalence should also be cautiously interpreted given the uncertainty in test characteristics. In particular, studies for the UK based on orders of magnitude more animals have suggested an exceptionally high specificity for the comparative test [55] of up to $100 \%$.

The tuberculin used in the two rounds of testing was not of the same batch although produced by the same company. This might have caused some discrepancy in the SICCT test results [56]; thus, in future studies, we would recommend the use of the same batch of tuberculin in multiple measurements, combining the SICCT test with other more sensitive test methods, such as the defined antigen skin test (DST) [57], to identify infected cattle with false-negative test results. We also recommend applying ear tags, such as ear notches or photographs of animals to mitigate the identification issues of cattle and the potential confounding that they may introduce.

\section{Farmers' response to disclosure of reactor status and potential impacts on control}

Despite the challenges in matching animals between tests, our supplemental questionnaire data demonstrate that some farmers choose to remove reactor animals from their herds, which was associated with a reduced risk of new reactors being detected to the herd over a comparatively short time-scale of 1-2 years.

This study shows that the voluntary removal of SICCT test reactor cattle by farmers would be a likely consequence of the survey implemented. Even though our analysis suggests this, it could lead to a reduction in the rate of transmission within these herds; however, if these animals are not slaughtered but sold to other herds, it could increase transmission between herds, given that there is no current legislative barrier to trading reactor animals. The Ethiopian government is currently considering establishing a systematic bTB surveillance programme in government-owned herds. Our results illustrate the need to carefully consider the regulation of sales of reactor animals as part of any initiative and the need for careful monitoring of the impacts as any nascent surveillance programme is rolled out.

In conclusion, the present study showed stable bTB apparent prevalence between surveys despite systematic and consistent differences in prevalence between regions. We found that the risk of transmission within herds was most strongly related to the age of animals and the within-herd apparent prevalence. Voluntary action towards the removal of reactor cattle should be encouraged, but there is currently a statutory gap with respect to preventing the potential increased risks of onward transmission between herds, which requires attention in the future.

Acknowledgements. The authors are indebted to the management of the National Animal Health Diagnostic and Investigation Centre (NAHDIC) and its technical staff who supported the field testing and data collection; Regional Agricultural Bureaus or Livestock Agencies, Dairy co-operations and Dairy farmers who collaborated or allowed this work to be done. We would also like to extend our appreciations to the editors and reviewers for their contribution to shape the manuscript.

Author contributions. GAM and AJKC conceived and designed the research, contributed equally to data analysis and write up. SB, JLNW, AM and GA conceived and designed the research; GAM, HA and AO performed the research; GAM, AJKC and BTA analysed the data; GAM and AJKC wrote the manuscript; SB, BTA, AM, GA and JLNW critically reviewed the manuscript.

Financial support. This work was funded by the Biotechnology and Biological Sciences Research Council, the Department for International Development, the Economic \& Social Research Council, the Medical Research Council, the Natural Environment Research Council and the Defence Science \& Technology Laboratory, under the Zoonoses and Emerging Livestock Systems (ZELS) programme, ref: BB/L018977/1. Stefan Berg was also funded by Defra, UK, ref: TBSE3294.

Conflict of interest. None.

Data availability statement. All data generated and/or analysed during the current study are not publicly available but are available from the corresponding author on reasonable request.

\section{References}

1. Ayele WY et al. (2004) Bovine tuberculosis: an old disease but a new threat to Africa. International Journal of Tuberculosis and Lung Disease 8, 924-937.

2. World Health Organization (2017) The Roadmap for Zoonotic Tuberculosis. Geneva, Switzerland: World Health Organization, International Organization for Animal Health, Food and Agriculture Organization, The Union. A Call to Action, 24p.

3. Ameni G, Bekele S and Tolosa T (2010) Preliminary study on the impact of bovine tuberculosis on the reproductive efficiency and productivity of 
Holstein dairy cows in central Ethiopia. Bulletin of Animal Health and Production in Africa 58, 223-228.

4. Caminiti A et al. (2016) Control and eradication of tuberculosis in cattle: a systematic review of economic evidence. Veterinary Record 179, $70-75$.

5. Humblet MF, Boschiroli ML and Saegerman C (2009) Classification of worldwide bovine tuberculosis risk factors in cattle: a stratified approach. Veterinary Research 40, 50.

6. Tschopp R et al. (2013) Cost estimate of bovine tuberculosis to Ethiopia. Current Topics in Microbiology and Immunology 365, 249-268.

7. Cosivi O et al. (1998) Zoonotic tuberculosis due to Mycobacterium bovis in developing countries. Emerging Infectious Diseases 4, 59-70.

8. de la Rua-Domenech $\mathbf{R}$ et al. (2006) Ante mortem diagnosis of tuberculosis in cattle: a review of the tuberculin tests, gamma-interferon assay and other ancillary diagnostic techniques. Research in Veterinary Sciences 81, 190-210.

9. Hamzi AE-I (2010) Bovine tuberculosis. In Mohd-Nor N, Acosta A and Sarmiento ME (eds), The Art and Science of Tuberculosis Vaccine Development, 2nd Edn. Selangor Darul Ehsan, Malaysia: Oxford Fajar Sdn Bhd, pp. 75-90.

10. Department of Agriculture, Fisheries and Forestry (2012) Australia's Freedom from Bovine Tuberculosis (TB). Canberra, Australia: Department of Agriculture, Fisheries and Forestry (DAFF), 4p.

11. Michel AL, Müller B and van Helden PD (2010) Mycobacterium bovis at the animal-human interface: a problem, or not. Veterinary Microbiology 140, 371-381.

12. Romha G et al. (2013) Epidemiology of mycobacterial infections in cattle in two districts of Western Tigray Zone, northern Ethiopia. African Journal of Microbiology Research 7, 4031-4038.

13. Amenu K et al. (2010) Brucellosis and tuberculosis in Arsi-Negele district, Ethiopia: prevalence in ruminants and people's behaviour towards zoonoses. Tropicultura 28, 205-210.

14. Tschopp $\mathbf{R}$ et al. (2010) Bovine tuberculosis at the wildlife-livestockhuman interface in Hamer Woreda, South Omo, Southern Ethiopia. PLoS ONE 5, e0012205.

15. Tschopp R et al. (2010) Repeated cross-sectional skin testing for bovine tuberculosis in cattle kept in a traditional husbandry system in Ethiopia. Veterinary Record 167, 250-256.

16. Gumi B et al. (2012) Low prevalence of bovine tuberculosis in Somali pastoral livestock, southeast Ethiopia. Tropical Animal Health and Production 44, 1445-1450.

17. Ameni G, Bonnet $\mathbf{P}$ and Tibbo M (2003) A cross-sectional study of bovine tuberculosis in selected dairy farms in Ethiopia. The International Journal of Applied Research in Veterinary Medicine 1, 253-258.

18. Tschopp R et al. (2013) Bovine tuberculosis and brucellosis prevalence in cattle from selected milk cooperatives in Arsi zone, Oromia region, Ethiopia. BioMed Central Veterinary Research 9, 163.

19. Sibhat B et al. (2017) Bovine tuberculosis in Ethiopia: a systematic review and meta-analysis. Preventive Veterinary Medicine 147, 149-157.

20. Firdessa R et al. (2012) High prevalence of bovine tuberculosis in dairy cattle in central Ethiopia: implications for the dairy industry and public health. PLOS ONE 7, e0052851.

21. Mekonnen GA et al. (2019) Network analysis of dairy cattle movement and its implication on associations with bovine tuberculosis spread and control in emerging dairy belts of Ethiopia. BioMed Central Veterinary Research 15, 262.

22. Tschopp R et al. (2012) Cost estimate of bovine tuberculosis to Ethiopia. Current Topics in Microbiology and Immunology 365, 249-268.

23. Shitaye JE et al. (2006) A prevalence study of bovine tuberculosis by using abattoir meat inspection and tuberculin skin testing data, histopathological and IS6110 PCR examination of tissues with tuberculous lesions in cattle in Ethiopia. Veterinarni Medicina 51, 512-522.

24. World Health Organization. Estimates of TB and MDR-TB Burden. Geneva: World Health Organization; 14 November 2018. Available at https:/extranet. who.int/sree/Reports?op=Replet\&name=/WHO_HQ_Reports/G2/PROD/ EXT/TBCountryProfile\&ISO2=ET\&outtype=PDF (Accessed 12 December 2019).
25. Müller B et al. (2013) Zoonotic Mycobacterium bovis-induced tuberculosis in humans. Emerging Infectious Diseases 19, 899-908.

26. Tibebu $\mathbf{M}$ et al. (2014) A high prevalence of tuberculosis among dairy farm workers in Addis Ababa and its surroundings. Journal of Mycobacterial Diseases 4, e1000139.

27. Otchere ID et al. (2019) Molecular epidemiology and whole genome sequencing analysis of clinical Mycobacterium bovis from Ghana. PLoS ONE 14, e0209395.

28. Gumi B et al. (2012) Zoonotic transmission of tuberculosis between pastoralists and their livestock in South-East Ethiopia. Ecohealth 9, 139-149.

29. Ameni G et al. (2007) Effect of skin testing and segregation on the incidence of bovine tuberculosis, and molecular typing of Mycobacterium bovis in Ethiopia. Veterinary Record 161, 782-786.

30. Central Statistical Agency (2015) City Population. Addis Ababa, Ethiopia: Central Statistical Agency. Available at https://www.citypopulation.de/ Ethiopia.html (Accessed 5 September 2019).

31. Mekonnen GA et al. (2019) Prevalence of bovine tuberculosis and its associated risk factors in the emerging dairy belts of regional cities in Ethiopia. Preventive Veterinary Medicine 168, 81-89.

32. Goodchild AV and Clifton-Hadley RS (2001) Cattle-to-cattle transmission of Mycobacterium bovis. Tuberculosis 81, 23-41.

33. International Organization for Animal Health (2018) Bovine tuberculosis. In: OIE Biological Standards Commission, ed. Manual of Diagnostic Tests and Vaccines for Terrestrial Animals. Paris, France: OIE, pp. 1058-1074.

34. Bates D et al. (2017) lme4: Linear Mixed-Effects Models using 'Eigen' and S4. R package, version 1.1-13.

35. Barr DJ et al. (2013) Random effects structure for confirmatory hypothesis testing: keep it maximal. Journal of Memory and Language 68, 255278.

36. Gelman A and Hill J (2007) Data Analysis Using Regression and Multilevel/ Hierarchical Models. Cambridge, UK: Cambridge University Press.

37. Lüdecke D, Makowski D and Waggoner P (2019) Performance: assessment of regression models performance, $\mathrm{R}$ package, version 0.4.2.

38. Lele SR, Keim JL and Solymos P (2017) ResourceSelection: probability functions for use-availability data. $\mathrm{R}$ package, version 0.3-2.

39. Robin X et al. (2017) pROC: display and analyze ROC curves. R package, version 1.10.0.

40. Lesnoff $\mathbf{M}$ and Lancelot R (2012) aod: Analysis of overdispersed data. R package, version 1.3.

41. Barnier J, Briatte F and Larmarange J (2017) questionr: Functions to make surveys processing easier. $\mathrm{R}$ package, version 0.6.2.

42. Sergeant ESG (2018) Epitools epidemiological calculators. Ausvet. Available at http://epitools.ausvet.com.au (Accessed 20 April 2017).

43. Dean AG, Sullivan KM and Soe MM (2013) OpenEpi: open source epidemiologic statistics for public health. Available at www.OpenEpi.com (Accessed 10 March 2020).

44. Reynolds D (2006) A review of tuberculosis science and policy in Great Britain. Veterinary Microbiology 112, 119-126.

45. Petukhov VL (1981) Genetics of cattle resistance to tuberculosis. The age of having the disease, milk productivity and the maternal influence on the incidence of the infection in progeny. Genetika 17, 1088-1094.

46. Benham PFJ (1985) A Study of Cattle and Badger Behaviour and Farm Husbandry Practices Relevant to the Transmission of Bovine Tuberculosis (Mycobacterium bovis). Reading, UK: Ministry of Agriculture, Fisheries and Food.

47. Francis J (1947) Bovine Tuberculosis: Including a Contrast with Human Tuberculosis. London: Staples Press Limited, 228 p.

48. More SJ and Good M (2015) Understanding and managing bTB risk: perspectives from Ireland. Veterinary Microbiology 176, 209-218.

49. Clegg TA et al. (2018) Trends and predictors of large tuberculosis episodes in cattle herds in Ireland. Frontiers in Veterinary Science 5, 86-86.

50. Goodchild A et al. (2015) Specificity of the comparative skin test for bovine tuberculosis in Great Britain. Veterinary Record 177, 258.

51. Karolemeas K et al. (2012) Estimation of the relative sensitivity of the comparative tuberculin skin test in tuberculous cattle herds subjected to depopulation. PLOS ONE 7, e0043217. 
52. Monaghan ML et al. (1994) The tuberculin test. Veterinary Microbiology 40, 111-124.

53. European Food Safety Authority (2012) Panel on animal health and welfare, scientific opinion on the use of a gamma interferon test for the diagnosis of bovine tuberculosis (European Food Safety Authority). European Food Safety Authority Journal 10, 2975.

54. Ameni G et al. (2008) Appraisal of interpretation criteria for the comparative intradermal tuberculin test for diagnosis of tuberculosis in cattle in central Ethiopia. Clinical and Vaccine Immunology 15, 1272-1276.
55. Nuñez-Garcia J et al. (2018) Meta-analyses of the sensitivity and specificity of ante-mortem and post-mortem diagnostic tests for bovine tuberculosis in the UK and Ireland. Preventive Veterinary Medicine 153, 94-107.

56. Downs SH et al. (2013) Tuberculin manufacturing source and breakdown incidence rate of bovine tuberculosis in British cattle, 2005-2009. Veterinary Record 172, 98-98.

57. Srinivasan S et al. (2019) A defined antigen skin test for the diagnosis of bovine tuberculosis. Science Advances 5, eaax4899. 